\title{
Blue Light-Induced Branching in Vaucheria. Requirement of Nuclear Accumulation in the Irradiated Region
}

\author{
Fumio Takahashi ${ }^{1,3}$, Tasuku Hishinuma ${ }^{2}$ and Hironao Kataoka ${ }^{1,4}$ \\ ${ }^{1}$ Institute of Genetic Ecology, Tohoku University, Aoba-ku, Sendai, 980-8577 Japan \\ ${ }^{2}$ Department of Biology, Faculty of Science, Yamagata University, Yamagata, 990-0021 Japan
}

When a narrow region of the fresh water coenocytic alga, Vaucheria terrestris sensu Götz is irradiated with moderately intense blue light, a branch is induced from the center of the irradiated region after 4-5 h. Movement of organelles and microtubule bundles during the photocytomorphogenetic response were investigated. Chloroplasts in the cortical layer immediately started to accumulate in the blue light-irradiated region and their accumulation almost completely finished 30-40 min after the onset of light when the nuclei residing in endoplasm started to accumulate. Accumulation of nuclei was synchronized with disorientation and shortening of microtubule bundles, which originally run parallel to the cell axis. Not only amiprophos-methyl, a potent microtubule-decomposing reagent, but also cytochalasin A strongly inhibited the branch induction. Amiprophosmethyl completely and cytochalasin A mostly destroyed microtubules and completely inhibited nuclear accumulation, but both drugs allowed the accumulation of chloroplasts in the cortical layer of irradiated region. These indicate that the accumulation of nuclei is indispensable for branch induction while the chloroplast accumulation is insufficient by itself for branch induction. The ineffectiveness of cytochalasin A on chloroplast movement brings the conventional view of sliding movement of chloroplast on a long actin cable into question. The morphological and functional relationship between a nucleus and a microtubular bundle are discussed.

Key words: Blue light - Branch induction - Coenocytes Microtubules — Nuclear movement - Vaucheria (Stramenopile).

Abbreviations: APM, amiprophos methyl, $N$-isopropyl $O$-methyl $O$-(2-nitro- $p$-tolyl) phosphoramidothioate; BL, blue light; BSA, bovine serum albumin; CA, cytochalasin A; DAPI, 4',6'-diamidino-2phenylindole dihydrochloride; DMSO, dimethylsulfoxide; FITC, fluorescein isothiocyanate; PBS, phosphate-buffered saline; PIPES, piperazine-1,4-bis(2-ethanesulfonic acid); MT, microtubule.

\section{Introduction}

Cytomorphogenesis is one of the fundamental functions of a plant cell necessary for growth and movement towards a suitable environment (Kiermayer 1981, Nagata 1973, Riquelme et al. 1998, Wada 1995). Of various cytomorphogenetic responses, light regulation of tip growth and branching seem to be of primary importance for understanding the spatial control of plant development. Giant coenocytic algae are particularly useful for study of photocytomorphogenesis, because they are easy to handle. Furthermore, it is of interest to compare behaviors and/or roles of nuclei in a coenocytic cell with multicellular organisms where morphogenesis is usually based on siterestricted cell division. Kataoka (1975b) first reported lightinduced branching in Vaucheria. When a part of the cylindrical coenocytic cell of Vaucheria geminata was irradiated with blue light (BL), one or two branches developed from the center of the BL-irradiated region 5-7 h after the onset of light. Only BL and white light, but not red or green light, was effective.

The body of Vaucheria consists of a sparsely branched coenocytic tube (Kataoka 1975a, Kataoka 1975b). Most of the intracellular space is occupied by central vacuole; chloroplasts are localized in the cortical layer lining the cell wall, and nuclei, in the inner cytoplasmic layer. Vaucheria does not form a septum except when it develops sexual organs and synzoospores, or when it is mechanically injured. When the coenocytic cell is injured and consequently segregated into fragments by its wound healing activity, a similar branch(es) develops from either one or both ends of the fragment. However, these are not true branches, because the cell continuum bearing such a branch is physically isolated from adjacent fragments. A true branch, which Vaucheria spontaneously develops and thus dealt with in this article, does not require any segregation wall.

The first visible intracellular event in Vaucheria caused by local BL-irradiation is accumulation of chloroplasts in the BL region. Senn (1908) first correlated the positioning of chloroplasts in relation to the intracellular distribution of light intensity. If low-intensity light was shone onto the cylindrical cell of Vaucheria from above, chloroplasts moved to the upper and lower walls, but with high-intensity light they moved to the side wall parallel to the path of light. Movement of chloroplast(s) towards the lighted wall of the plant cell has been defined as low-fluence-rate (orientation) movement (Haupt 1963, Haupt 1965, Haupt and Schönbohm 1970). Besides

\footnotetext{
${ }^{3}$ Present address: Photodynamics Research Center, The Institute of Physical and Chemical Research (RIKEN), Aramaki, Aoba-ku, Sendai, 9800845 Japan.

${ }^{4}$ Corresponding author: E-mail, kataoka@ige.tohoku.ac.jp; Fax, +81-22-263-9845.
} 
transversal movement, chloroplasts also move longitudinally into lighted region. Since the chloroplast is not a motile organelle, it must be passively driven by cytoplasmic motor system. By means of a microbeam irradiation method FischerArnold (1963) provided evidence of the photoreceptor for chloroplast accumulation in Vaucheria being not in the chloroplast but in the cortical cytoplasm layer.

Using a vibrating electrode method, Blatt and his colleagues found that the BL-induced accumulation of chloroplasts of $V$. sessilis was associated with an efflux of ionic current from the BL-irradiated region (Blatt and Briggs 1980a, Blatt et al. 1981). The nature of the outward current was suggested to be BL-induced proton extrusion at the plasma membrane (Blatt et al. 1981). The action spectrum for the chloroplast accumulation of Vaucheria had a single peak at around $470 \mathrm{~nm}$ and an increase in the UV-A region (Blatt 1983). They observed that the cortical cytoplasmic cables, probably being composed of actin filaments, were reticulated in the BLirradiated region and hypothesized that chloroplasts are moved along the actin filaments (Blatt and Briggs 1980b, Blatt et al. 1980).

The outward current was, however, stopped about $1 \mathrm{~h}$ after the onset of light and a very large inward current began at the irradiated site after $2 \mathrm{~h}$ (Kicherer 1985). This large inward current continued until a branch was formed, and if the inward current stopped, no branch was induced. This was the first indication that chloroplast accumulation and induction of branching are kinetically different responses. Since the current influx at the apex of Vaucheria is stimulated by BL (Kataoka and Weisenseel 1988), the preceding inward current at the BL-irradiated site can be regarded as a measurable sign of the initiation process of the branch.

The aim of the present study was to determine essential intracellular events of the BL-induced cytomorphogenetic response and to understand the sequential regulation pathways leading to branching.

\section{Materials and Methods}

\section{Plant material and culture}

The fresh water species, V. terrestris sensu Götz, used in the previous studies (Kataoka 1981, Kataoka 1987, Kataoka 1988, Kataoka 1990, Kataoka and Watanabe 1993), was also used in this study. The culture medium was slightly modified from that previously used (Kataoka 1981) in that the new medium was supplemented with $200 \mathrm{ng} \operatorname{liter}^{-1}$ thiamin and $48 \mathrm{nM} \mathrm{CuSO}$. Other culture conditions were essentially the same as before (Kataoka 1981).

\section{Preparation of cell segments for branch induction}

Pieces of thalli were pinched off from the preculture and incubated in a Petri dish at $20^{\circ} \mathrm{C}$ under white fluorescent lamps at a fluence rate of approximately $15 \mu \mathrm{mol} \mathrm{m} \mathrm{m}^{-2} \mathrm{~s}^{-1}, 12 \mathrm{~h}$ light/12 h dark regimens for $7 \mathrm{~d}$. Long, straight, non-branched thalli were selected and cut into about 2-cm-long segments with fine oculist scissors. These segments were further cultured in plastic dishes for another $2 \mathrm{~d}$. Since Vaucheria is characterized by very high wound healing activity and extremely low water permeability (Tazawa and Kataoka, unpublished), plasmoptysis does not take place and the segments regain their normal osmotic pressure within $2 \mathrm{~d}$. Nevertheless, because these segments had lost their turgor and were very sensitive to mechanical shock immediately after cutting, great care was taken not to bend the segments. Thus, upon transfer, every segment was gently sucked into a Pasteur pipette under a stereo microscope and slowly pushed out into the new culture vessel.

Two days after the transfer, 3 to 4 appropriate segments, i.e. straight, healthy looking, having no segregation walls within the continuum and possessing a single growth center at one end, were selected and placed in a $6-\mathrm{cm}$ plastic dish. The segments were laid parallel to each other with the growing apices oriented in the same direction. Proximal ends of the segments were, if necessary, fixed with drops of agar ( $1 \%$ low gelling temperature, $30-31^{\circ} \mathrm{C}$, Nacalai Tesque, Kyoto, Japan), and the dishes were filled with culture solution and placed in a dark room $\left(20^{\circ} \mathrm{C}\right)$ for another day.

\section{Optics employed for the branch induction}

The optical setup used was essentially the same as that for splitfield illumination (Kataoka 1975a, Kataoka 1980). Two heat-cut filters (IRA25S, Toshiba-Kasei, Tokyo) and an interference filter $\left(\mathrm{T}_{\max }=\right.$ $456 \mathrm{~nm}$, spectral full width at half maximum $=17 \mathrm{~nm}$, KL-45, Toshiba-Kasei) were inserted just after the field stop of the microscope illuminator. A slit made of razor blades was placed near the field stop. A thin glass plate was inserted at $45^{\circ}$ between the slit and the microscope-condenser to illuminate the specimen with dim red observation light $\left(\mathrm{T}_{\max }=660 \mathrm{~nm}, \mathrm{KL}-66+3 \mathrm{~mm}\right.$ red acrylic plate, Acrilyte \# 102, Mitsubishi Rayon, Tokyo; fluence rate $<0.3 \mathrm{~W} \mathrm{~m}^{-2}$ ).

The image of the slit was focused at the specimen level, so as to satisfy the condition of Köhler illumination. The incandescent-lamp microscope-illuminator glowed at $4 \mathrm{~V}$ (from a stabilized DC supply, GM08-10, Kawasaki, Japan). The fluence rate of the 456-nm light (BL) at the specimen level was $1.95 \mathrm{~W} \mathrm{~m}^{-2}\left(7.3 \mu \mathrm{mol} \mathrm{m} \mathrm{m}^{-2} \mathrm{~s}^{-1}\right)$ at $4 \mathrm{~V}$, as measured by a calibrated custom-made photometer-amplifier equipped with a silicon photocell (S1226-8BQ, Hamamatsu Photonics, Japan). All light experiments were conducted in a dark room at $20^{\circ} \mathrm{C}$ under dim red light. A specimen prepared as above was placed on the stage of a microscope and the image of the slit, 50-500 $\mu \mathrm{m}$ in width, was focused on the middle plane of the Vaucheria cell; irradiation was continued until branches were induced. The presence of dim red observation light had no effect (Kataoka 1975b, Kataoka 1981). A $4 \times$ objective was used to observe the alga through the lid of the dish. A charge-coupled device (CCD) video camera (XC-77; Sony, Atsugi, Japan) and videocassette recorder (Panasonic AG6720A; National, Osaka, Japan) were used for the measurement of branching. For taking photographs (Fig. 1, 5, 11), a similar optical setup was constructed with an Olympus BH microscope equipped with a halogen-tungsten lamp and a $10 \times$ objective (SPlan $10, \mathrm{NA}=0.30$ ). A broadband blue glass filter, instead of interference filter, was used for color photographs.

\section{DAPI staining}

Distribution of nuclei was observed fluorescence microscopically after staining with $4^{\prime}, 6^{\prime}$-diamidino-2-phenylindole, dihydrochloride (DAPI). Specimens treated with local BL irradiation for 0 to $7 \mathrm{~h}$ were immediately fixed in the dark with $1 \%$ glutaraldehyde dissolved in phosphate-buffered saline (PBS; $137 \mathrm{mM} \mathrm{NaCl}, 2.7 \mathrm{mM} \mathrm{KCl}$, $\left.1.5 \mathrm{mM} \mathrm{KH} \mathrm{PO}_{4}, 8 \mathrm{mM} \mathrm{NaH} \mathrm{PO}_{4}, \mathrm{pH} 7.3\right)$ for $1 \mathrm{~h}$. After thorough washing with $\mathrm{PBS}$, segments including BL-irradiated regions were isolated with fine scissors and treated with $1 \%$ Triton X-100 to extract chlorophyll which would have otherwise resulted in strong autofluorescence; the specimens were then washed several times with PBS to 
eliminate the detergent. Specimens were then stained with $1 \mathrm{mg} \operatorname{liter}^{-1}$ DAPI for $30 \mathrm{~min}$, washed, and mounted on slide glass with or without glycerin : PBS $(1: 1)$ solution. Epifluorescence microscopes (Labophoto + EFD-2, Nikon, Japan; BH-2 + BH2-RFC, Olympus, Japan; Axioplan, Carl Zeiss, Germany) were used. Analysis was carried out on photographic prints.

\section{Immunofluorescence microscopy for microtubules}

Methods developed by Kadota and Wada (1992) were modified for the immunofluorescence detection of microtubules. A mixture of $1.8 \%$ paraformaldehyde (Nacalai Tesque) and $0.2 \%$ glutaraldehyde (Nacalai Tesque) in a PIPES buffer $(\mathrm{pH} 7.0)$ that contained $5 \mathrm{mM}$ ethyleneglycol-bis-( $\beta$-aminoethylether)- $N, N, N^{\prime}, N^{\prime}$-tetraacetic acid, $5 \mathrm{mM}$ $\mathrm{MgCl}_{2}, 0.5 \mathrm{mM}$ phenylmethylsulfonyl fluoride, $1 \%$ dimethylsulfoxide, and $10 \mathrm{mM}$ piperazine-1,4-bis(2-ethanesulfonic acid) (PIPES, Dojin, Kumamoto) was used for fixation (at room temperature for $2 \mathrm{~h}$ ). During the fixation, the algal filament was carefully cut into segments. After being washed with the PIPES buffer for $20 \mathrm{~min}$, segments about $500 \mu \mathrm{m}$ long that contained the BL-irradiated region were transferred to a hollow glass plate filled with the PIPES buffer. Subsequent procedures for immunostaining were carried out on this plate.

Pretreatment with $1.0 \%$ Triton X-100 to permeabilize the plasmalemma and chloroplast membrane, which was necessary for reducing autofluorescence, was performed by gentle perfusion with a fine pipette. Since Vaucheria membrane is less permeable to chemicals and segments are sometimes clotted by a mass of protoplasm, this gentle perfusion was also extraordinarily helpful for quick penetration of antibodies. To reduce non-specific binding with antibodies, specimens were blocked with $0.1 \%$ bovine serum albumin (BSA, Sigma) in PBS buffer at $36^{\circ} \mathrm{C}$ for $1 \mathrm{~h}$, then stained with anti-chick brain- $\beta$-tubulin mouse monoclonal antibody (Amersham, Buckinghamshire, U.K.) $\left(1 / 200\right.$ dilution in PBS-BSA) at $36^{\circ} \mathrm{C}$ for $3-12 \mathrm{~h}$. After being washed with PBS-BSA, the specimens were labeled with fluorescein dye or Alexa 488 conjugated anti-mouse IgG goat antibody (N 1031, Amersham, or A11001, Molecular Probes, Leiden, The Netherlands) at the same temperature for 3-12 h. Double staining with DAPI sometimes followed this step, and in some cases, $0.1 \%$ paraphenylene diamine (Tokyo Kasei, Tokyo) was added to the mounting glycerin solution to prevent quick decay of fluorescence.

\section{Electron microscopy}

Vaucheria filaments were pre-fixed with $2.0 \%$ glutaraldehyde in Na-MOPS buffer [10 mM 3-morpholinopropanesulfonic acid (MOPS) and $5 \mathrm{mM} \mathrm{CaCl}_{2}$, pH being adjusted with $\mathrm{NaOH}$ to 7.0 ] at room temperature for $12 \mathrm{~h}$. After the pre-fixation, the filaments were cut into several pieces, and post-fixed with $1.0 \% \mathrm{OsO}_{4}$ in the Na-MOPS buffer for $6 \mathrm{~h}$. Specimens were dehydrated with a graded acetone series and embedded in Spurr's resin (Spurr 1969). Sections were stained with uranyl acetate and Reynolds' lead citrate solution (Reynolds 1963), and observed with a JEM100 SX (JEOL, Tokyo) electron microscope.

\section{Chemicals}

Amiprophos-methyl (APM), donated by Nihon Bayer Agrochem (Yuki, Japan; Aya et al. 1975) was dissolved at $1 \mathrm{mM}$ in $1 \%$ DMSO. Cytochalasin A (CA) was purchased from Aldrich Chem. (Milw. WI, U.S.A.), cytochalasin B (CB) was from I.C.I. Res. Lab. (Cheshire, U.K.), and cytochalasin C and D (CC, CD) were from Sigma (St. Louis, MO, U.S.A.). Cytochalasins were dissolved in dimethylsulfoxide (DMSO) at $10 \mathrm{mM}$ and stored in refrigerator until use. They were diluted with culture solution for use. One percent DMSO had no detectable effect on morphology and branching. Colchicine, colcemid and vinblastine sulfate were obtained from Sigma. Every chemical was added to the cultures at least $2 \mathrm{~h}$ prior to the irradiation experiments.

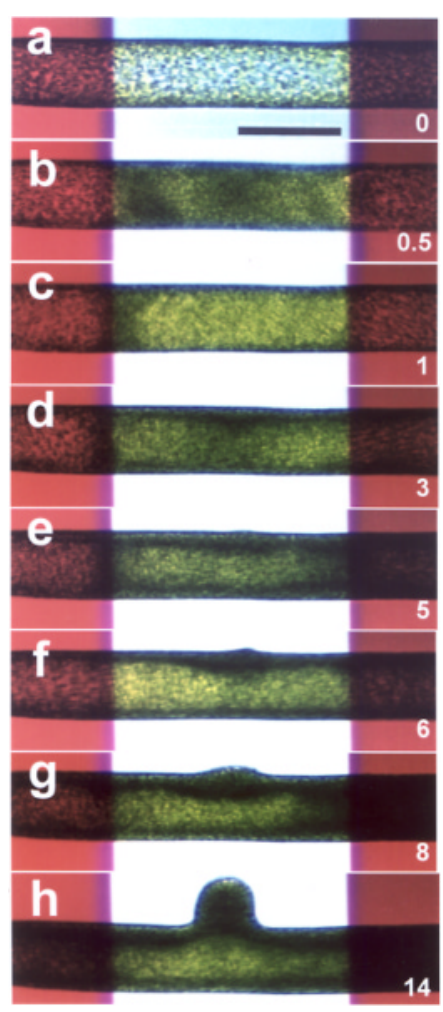

Fig. 1 Color photographs showing the time course of the BL-induced branching. A $225-\mu \mathrm{m}$ region of the proximal part of the cell was exposed to broadband BL (approximately $7 \mathrm{~W} \mathrm{~m}^{-2}$ ) for $14 \mathrm{~h}$. a, 0 min after the onset of BL; b, 30 min; c, 1 h; d, 3 h; e, 5 h; f, $6 \mathrm{~h} ; \mathrm{g}, 8 \mathrm{~h}$; h, $14 \mathrm{~h}$. Chloroplasts started to accumulate in the BL-irradiated region immediately after the onset of BL. After $1 \mathrm{~h}$, the thickness of the protoplasmic layer began to increase (c). Protoplasm continuously flowed into the BL region and the maximum thickness was found at the center of the BL-irradiated region (d). At $5 \mathrm{~h}$ a transparent patch appeared at the flank of the cell at the center of the BL region (e), which then grew into a protrusion (f, $g, h$ ) during subsequent $h$.

\section{Results}

\section{Time course of BL-induced branching}

Figure 1 shows an example of the BL-induced branching. Under diffuse light or in darkness, chloroplasts were equally distributed in the thin cortical cytoplasmic layer lining the interior of the cylindrical Vaucheria cell (Fig. 1a). They rushed into the $\mathrm{BL}$ region from adjacent dark regions after a short ( $<1 \mathrm{~min})$ lag, and a layer of chloroplasts completely occupied the cortical protoplasmic layer after $30 \mathrm{~min}$ (Fig. 1b). Movement of chloroplasts into the BL region often occurs in waves (Fig. 1c). This rapid chloroplast accumulation is restricted to cortical cytoplasmic layer and completes until $1 \mathrm{~h}$. The process probably coincides with the efflux of ionic current from the BLirradiated region (Blatt et al. 1981, Kicherer 1985). Kinetically different accumulation movement follows later on this rapid response. Inner protoplasm (=endoplasm) that contains chloro- 


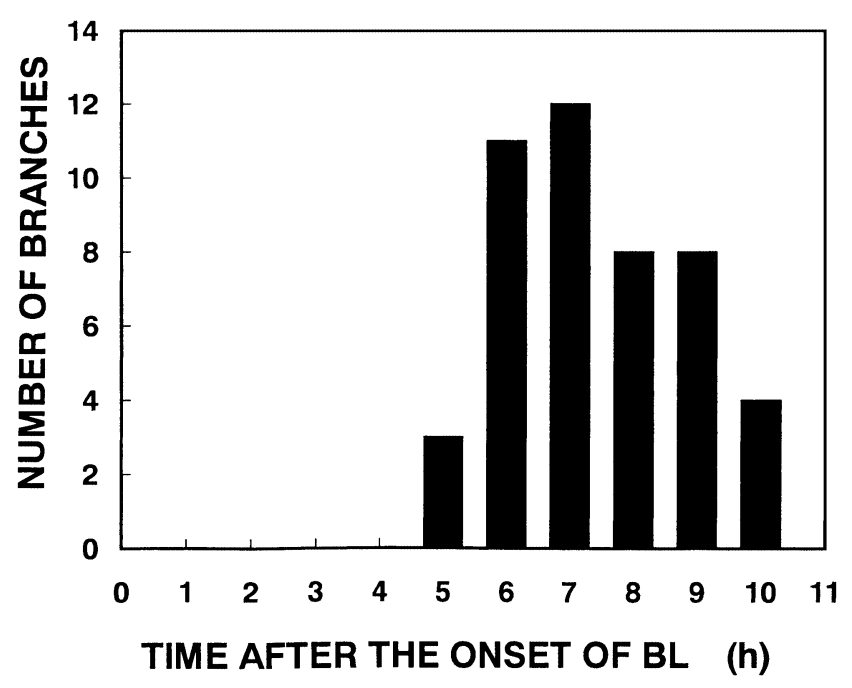

Fig. 2 Irradiation time required for the initiation of branching. Time of branching as determined by the appearance of small protrusions (as seen in Fig. 1e) is demonstrated. From a total of 70 cells subjected to the irradiation experiment (width of BL region, $200 \mu \mathrm{m}$; fluence rate of the $456 \mathrm{~nm}$-light, $1.95 \mathrm{~W} \mathrm{~m}^{-2}$ ), 46 cells developed branches within $10 \mathrm{~h}$, and 15 other cells, within $24 \mathrm{~h}$. Namely, $87 \%$ of the cells developed branches within $24 \mathrm{~h}$.

plasts, nuclei and other organelles starts to migrate into the BLirradiated region 40-60 min after the onset of light. Many chloroplasts sunk down to the tonoplast (seen as dark green lines in photographs, Fig. 1d-g). Thus, the accumulation of chloroplasts in the BL-irradiated region seems to proceed in two separate steps.

After 3-4 h, thickness of the protoplasm reached 10$25 \mu \mathrm{m}$ (Fig. 1e). A transparent patch developed at or near the center of the BL-irradiated area $4 \mathrm{~h}$ after the onset of light at the earliest (Fig. 1e), and this patch then bulged up to form a protrusion (Fig. 1f, g), which subsequently grew as a branch (Fig. 1h). This process was very similar to that previously described in V. geminata (Kataoka 1975b). Figure 2 shows that branches developed between 5 and $10 \mathrm{~h}$ after the onset of local BL-irradiation. Nonetheless, the time required for the branch initiation varied cell to cell and was not so closely dependent on the fluence rate, unless the fluence rate was too high at the point where chloroplasts moves out of the irradiated region (high fluence rate movement), resulting in branching from the margin(s) of the BL region or no branch induction.

Electron micrographs (Fig. 3) of the cross-section of the BL-irradiated region demonstrate a thick accumulation of protoplasm. The controls (Fig. 3a, b), taken after the cell was kept in darkness for $24 \mathrm{~h}$, demonstrate a dark arrangement of chloroplasts. Chloroplasts are distributed equally in the cortical layer of the cytoplasm. Surfaces of flat ellipsoidal chloroplasts are oriented parallel to the cell surface (face orientation) (Fig. $3 b)$. Figures $3 c$ and $d$ are the cross-section of the cell in the BLirradiated region $3 \mathrm{~h}$ after the onset of BL. We found a very

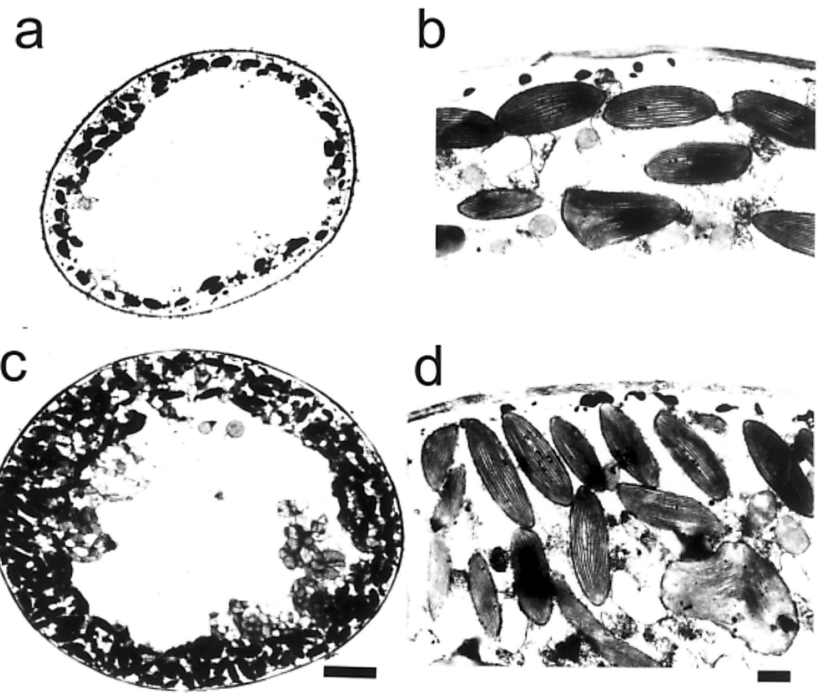

Fig. 3 Electron micrographs of the cross section of V. terrestris sensu Götz before and after BL irradiation. (a). Before BL irradiation (control). Cell was kept in darkness for $24 \mathrm{~h}$. (b). Magnification of a. Chloroplasts are horizontally oriented in 1-2 layers. (c). A $200-\mu \mathrm{m}$-width region irradiated with $\mathrm{BL}\left(456 \mathrm{~nm}, 1.95 \mathrm{~W} \mathrm{~m}^{-2}\right)$ for $3 \mathrm{~h}$. Thickness of the protoplasmic layer reaches $10-15 \mu \mathrm{m}$. Vacuolar inclusions are also transported into the BL region. (d). Magnification of c. Chloroplasts in the radial direction are piled up in 3-4 layers. Bars in a and c represent $10 \mu \mathrm{m}$. Bars in $\mathrm{b}$ and $\mathrm{d}$ represent $1.0 \mu \mathrm{m}$.

interesting phenomenon: besides the difference in thickness of protoplasm, the surfaces of the chloroplasts became perpendicular to the cell surface (profile orientation) $3 \mathrm{~h}$ after the onset of BL. With this rotation chloroplasts accumulated in the endoplasm would be able to receive light and perform photosynthesis. The mechanism of the chloroplast rotation is not known.

\section{Positional control of the BL-induced branching}

Branch induction usually occurs from the center of the BL-irradiated region (Fig. 1), unless the fluence rate of $\mathrm{BL}$ exceeds several $\mathrm{Wm}^{-2}$. The histogram (Fig. 4a) reveals that the branching occurred at the highest frequency from the center of the $\mathrm{BL}$ region. If the width of the $\mathrm{BL}$ region was as narrow as $50 \mu \mathrm{m}$ (Fig. 4b), a branch developed not only from the BL region but also from the adjacent dark area. In the case of Fig. $4 \mathrm{~b}$, as many as $40 \%(14 / 35)$ of the branches developed from the adjacent dark area. Both histograms, very similar in shape, are normal distributions with identical F-values. This means that the width of the $\mathrm{BL}$ region is not the parameter used to determine the site of branch initiation. A branch(es) was always formed from the site where the protoplasmic layer was the thickest. This suggests that the BL-induced products include, at least, the cellulolytic enzyme(s) that solubilize preexisting cell walls and substances that build a new growth center, and that these substances act in loco. This possibility was further supported by results of an experiment in which the 

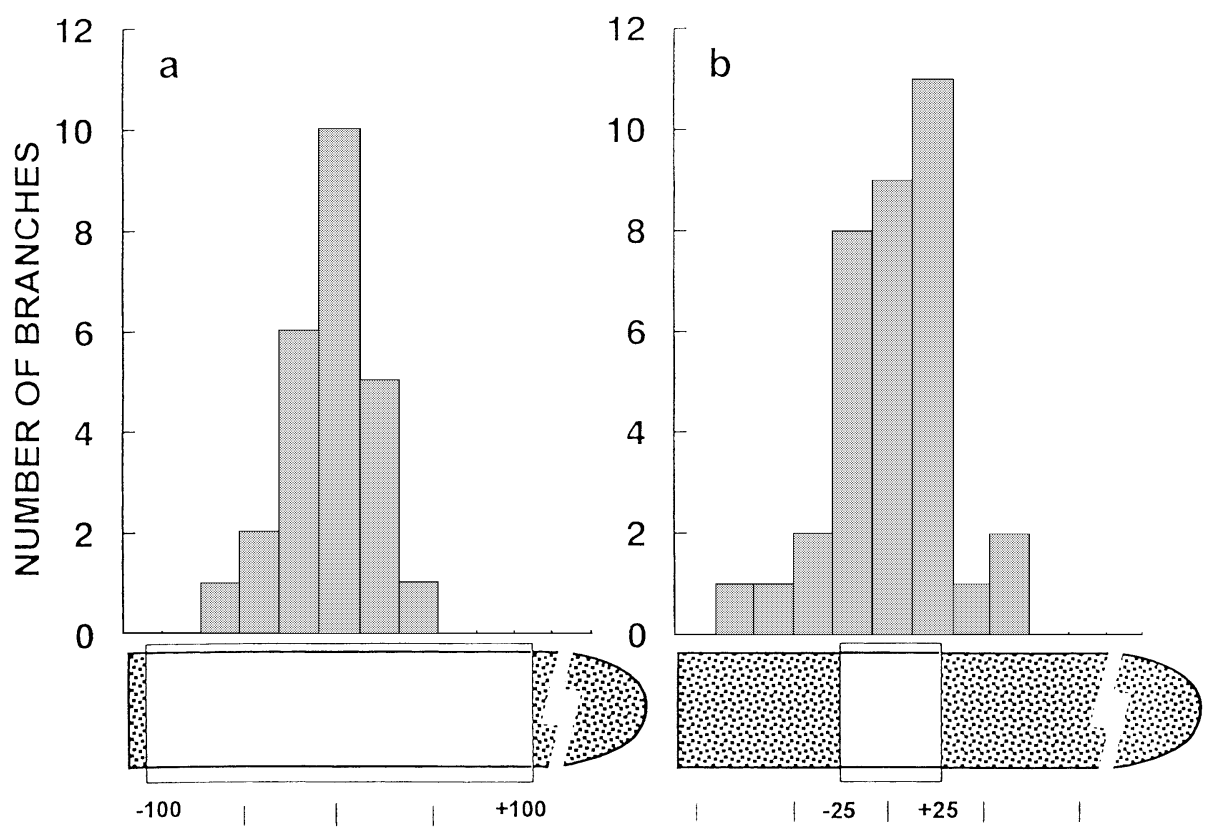

Fig. 4 Position of the branch relative to the BL region. Histograms showing the frequencies of the midpoints of the protrusions located in every $20-\mu \mathrm{m}$ fraction along the cell axis. BL source was the same as in Fig. 3. (a) Width of BL region: $200 \mu \mathrm{m}$. Branch develops at the highest probability from the center $\pm 10 \mu \mathrm{m}$. (b) Width of BL region: $50 \mu \mathrm{m}$. Branching equally occurs from the center and the margins of the BL region. A considerable number of branches (13 out of 35) develop from adjacent dark areas. Both a and $\mathrm{b}$ histograms are regarded as being normal distributions (a: $\chi^{2}=2.282, p=0.131$. b: $\chi^{2}=1.683, p=0.431$ ) of identical variance ( $\mathrm{F}$ tested, $\left.p=0.174\right)$.

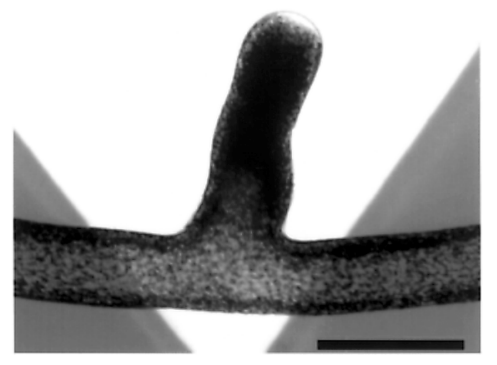

Fig. 5 BL-induced branching from a wedge-shaped BL beam. Proximal region of the cell was exposed to broadband BL (about $7 \mathrm{~W} \mathrm{~m}^{-2}$ ) for $12 \mathrm{~h}$. A branch developed from the center of the upper side of the light wedge. The diameter of the branch increased as it had been irradiated with strong BL (see Kataoka 1981). Bar $=100 \mu \mathrm{m}$.

area on one side of the cylindrical cell which received BL was wider than such area on the other side; a wedge-shaped BLbeam was used (Fig. 5). Branching always occurs from the more widely irradiated side.

Table 1 shows the relationship between the number and position of the branch(es) and the width of the BL-irradiated band at a fluence rate of $1.95 \mathrm{~W} \mathrm{~m}^{-2}$. Even when the width of the BL region was changed from $50 \mu \mathrm{m}$ to $500 \mu \mathrm{m}$, the frequency of branch induction did not change significantly, scoring a considerably high value (>70\%). However, when the width of the BL region was $50 \mu \mathrm{m}$ or wider than $200 \mu \mathrm{m}$, two branches occasionally developed from the irradiated region.

Accumulation of microtubule bundles and nuclei in the $B L$ irradiated region

Figure 6 demonstrates changes in density of nuclei and orientation of microtubule (MT) bundles during the BLinduced branching. The MT bundles in the cell dark-adapted for $1 \mathrm{~d}$ are observed to be thick, straight bundles, $50-70 \mu \mathrm{m}$ in length, mostly oriented parallel to the cell axis underneath the chloroplast layer of the coenocytic cells (Fig. 6a). They look much straighter but sparser than those observed in the green algal coenocyte Dichotomosiphon (Maekawa and Nagai 1988). Besides these long thick bundles, however, shorter and slightly undulating fine bundles extending obliquely to the cell axis are also discernible. However, there is no so-called cortical MT which are widely seen in diffuse growing cells.

Nuclei in the same region appear to be uniformly distributed in the thin endoplasmic layer (Fig. 6b). The density and the distribution of the nuclei seem to coincide with those of MT bundles. A $200-\mu \mathrm{m}$-wide area, usually several millimeters proximal to the growing apex, was irradiated with BL $(456 \mathrm{~nm})$ of $1.95 \mathrm{~W} \mathrm{~m}^{-2}$. The MT bundles running parallel to the cell axis became less clear $1 \mathrm{~h}$ after the onset of BL (Fig. 6c). Since the amorphous cloud emitted bright green fluorescence, MT bundles were probably disintegrated into monomeric tubulin. 
Table 1 Relationship between number of branches and width of BL-irradiated region

\begin{tabular}{lllll}
\hline BL-width & $50 \mu \mathrm{m}$ & $100 \mu \mathrm{m}$ & $200 \mu \mathrm{m}$ & $500 \mu \mathrm{m}$ \\
No. of cells used & 21 & 25 & 41 & \multicolumn{1}{l}{13} \\
\hline One branch & $16(76.2 \%)$ & $18(72.0 \%)$ & $29(70.7 \%)$ & $7(53.8 \%)$ \\
Two branches & $1(4.8 \%)$ & 0 & $6(14.6 \%)$ & $3(23.1 \%)$ \\
Total & $17^{a}(80.9 \%)$ & $18(72.0 \%)$ & $35(85.4 \%)$ & $10(76.9 \%)$ \\
\hline
\end{tabular}

Fluence rate of the $456 \mathrm{~nm}$ light: $1.95 \mathrm{~W} \mathrm{~m}^{-2}$. Branches were counted $24 \mathrm{~h}$ after the onset of light

${ }^{a} 11$ Branches were from adjacent dark area.

Although the MT bundles seemed to be shortened, thinned and disoriented in the BL region, the number of bundles rather increased. After $5 \mathrm{~h}$, randomly oriented MT bundles increased in density in the BL region (Fig. 6e), and after a branch developed $(10 \mathrm{~h})$, thick MT bundles became elongated again and reoriented towards the apex of the new branch (Fig. 6g). In the meantime, nuclei accumulated in the BL-irradiated region concomitantly with the MT bundles (Fig. 6d, f, h).
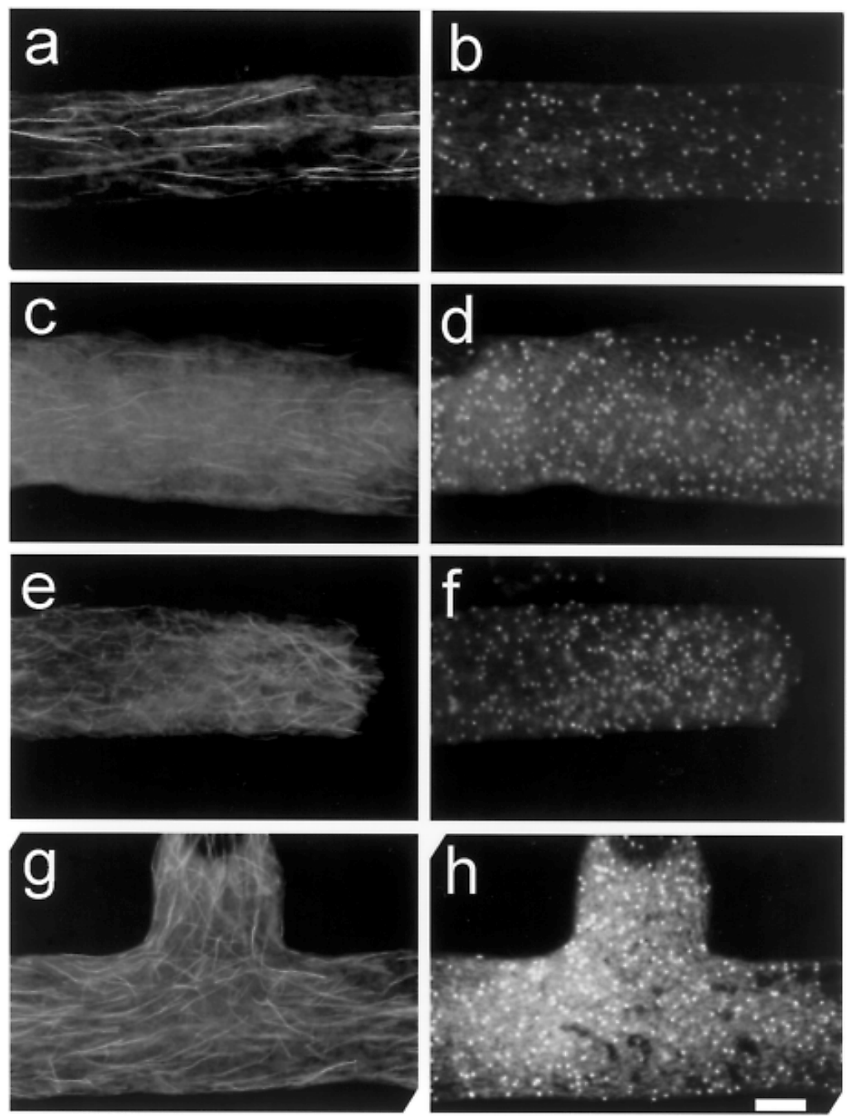

Fig. 6 Changes in orientation and distribution of MT bundles and nuclei during the course of BL-induced branching. Specimens were double stained with $\beta$ tubulin antibody and DAPI. Left sides: MT bundles; right sides: nuclei. A $200-\mu \mathrm{m}$ region was irradiated with BL $\left(456 \mathrm{~nm}, 1.95 \mathrm{~W} \mathrm{~m}^{-2}\right)$ for $0 \mathrm{~h}(\mathrm{a}, \mathrm{b}), 1 \mathrm{~h}(\mathrm{c}, \mathrm{d}), 5 \mathrm{~h}(\mathrm{e}, \mathrm{f})$ and $10 \mathrm{~h}(\mathrm{~g}$, h). $\mathrm{Bar}=20 \mu \mathrm{m}$.
As shown in magnified photographs (Fig. 7), until $1 \mathrm{~h}$ after the onset of BL, the orientation of MT bundles in the outer cytoplasmic layer (Fig. 7a) remained parallel to the cell axis, whereas in the inner endoplasm (Fig. 7b) thin and short MT bundles ran obliquely. By this time, 2-3 layers of chloroplasts had already filled the outermost layer of protoplasm. Figure 7 indicates that accumulation of nuclei and disorientation
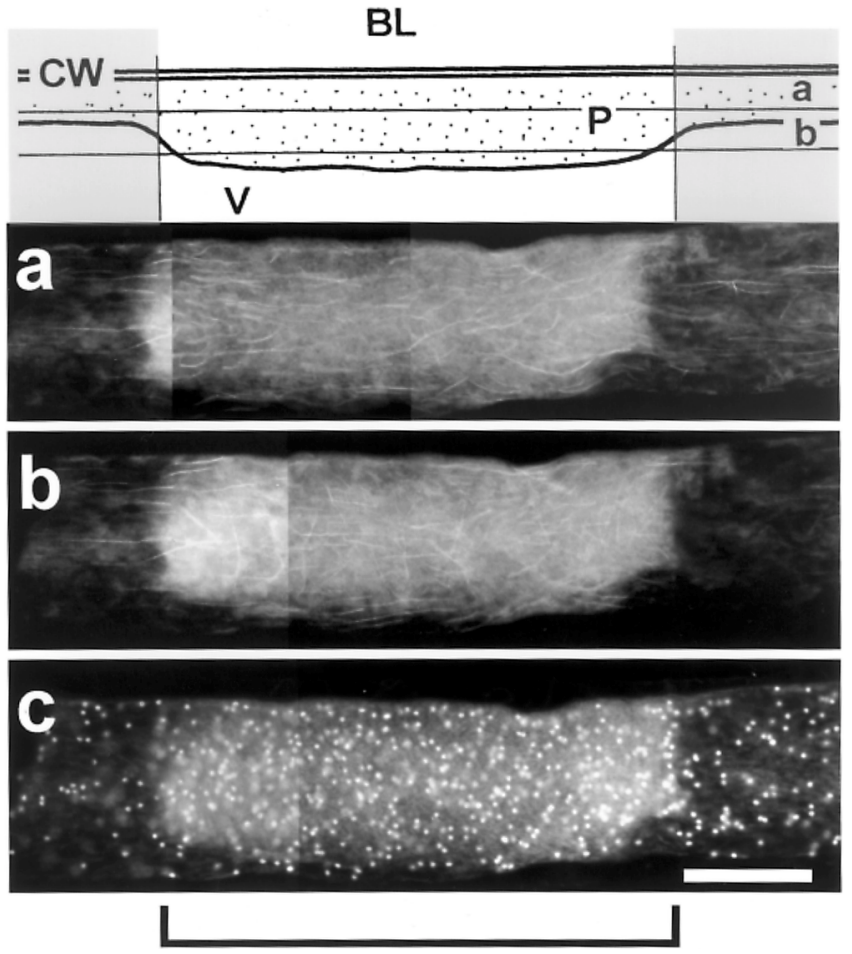

Fig. 7 Magnified photographs showing accumulation of MT bundles and nuclei $1 \mathrm{~h}$ after the onset of BL. The same specimen as in Fig. 6c, $\mathrm{d}$. The focal planes for the photographs are indicated in the sketch. (a) Parallel oriented MT bundles dominated in the peripheral layer (layer a) of protoplasm. Fluorescence from monomeric tubulin molecules, as the degradation product of MT bundles, and cytoplasm out of focus gave bright green fluorescence (gray areas). (b) Shorter MT bundles run obliquely in the inner protoplasmic layer (layer b). (c) Nuclei are accumulated in the inner protoplasmic layer (layer b) in the BL-irradiated region. Blue fluorescence from chloroplast- and mitochondrial DNA gave strong halation in BL-irradiated region. Bracket at the bottom of $\mathrm{c}$ indicates the region of $\mathrm{BL}$ irradiation. $\mathrm{Bar}=50 \mu \mathrm{m}$. 


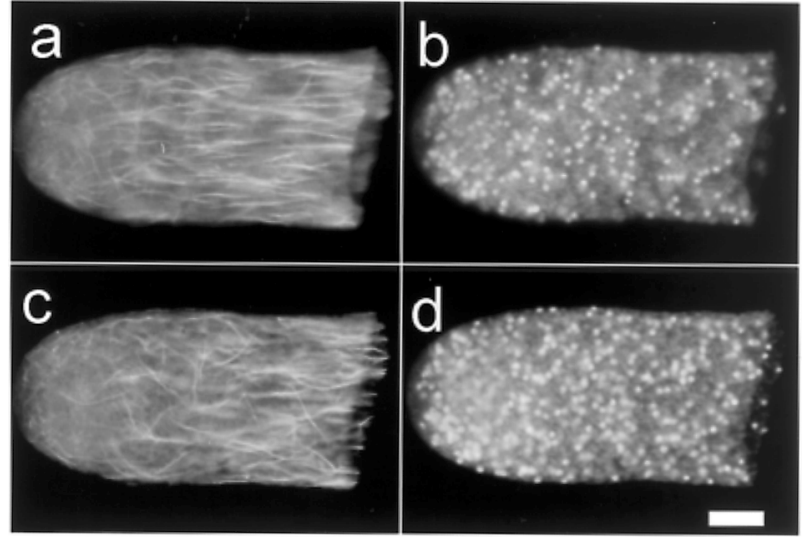

Fig. 8 MT bundles and nuclei in the apical region. A representative example is shown. Pairs, a and b, c and d, are photographs taken at the peripheral and middle layers of the single actively growing apex, respectively. MT bundles (a and c); Nuclei (b and d). Bar $=20 \mu \mathrm{m}$.

and degradation of MT bundles took place first in the inner endoplasmic layer about $1 \mathrm{~h}$ after the onset of BL. The degradation and/or disorientation of MT bundles then propagated to outer protoplasmic layer (Fig. 6e).

Orientation of MT bundles and distribution of nuclei in the newly developing branchlet resemble those observed in the growing apices of the main axis (Fig. 8). In the peripheral layer of the apical dome of a growing apex (Fig. 8a), a considerable number of MT bundles extend towards the very tip. In the core protoplasmic mass (Fig. 8c), however, there are clusters of short, fine MT bundles obliquely and/or perpendicularly oriented to the cell axis. Except at the very tip region, the densities and the distribution of nuclei coincide with those of MT bundles (Fig. 8b, d). The core region of the apex where there are many nuclei, emits bright green fluorescence (Fig. 8a, c). Since the region corresponds to apical transparent cap in which exocytotic vesicles are packed, the amorphous green fluorescence is probably due to monomeric tubulin pool. The amorphous green fluorescence is lacking below apical hemispherical dome (see also Fig. 6a, g).

Movement of nuclei into the BL-irradiated region is graphically demonstrated in Fig. 9. The number of nuclei per unit area $\left(2,500 \mu \mathrm{m}^{2}=50 \times 50 \mu \mathrm{m}\right)$ increased by the factor of two by $5 \mathrm{~h}$ in the BL-irradiated region, whereas that in the adjacent dark area decreased. At about 60 min when the rapid chloroplast accumulation in the cortical cytoplasmic layer had already finished, the nuclei started to accumulate. The possibility that the increase in the number of nuclei in the BLirradiated region was due to BL-triggered synchronous mitosis was excluded by careful observation of more than 200 specimens fixed at intervals of $30 \mathrm{~min}$.

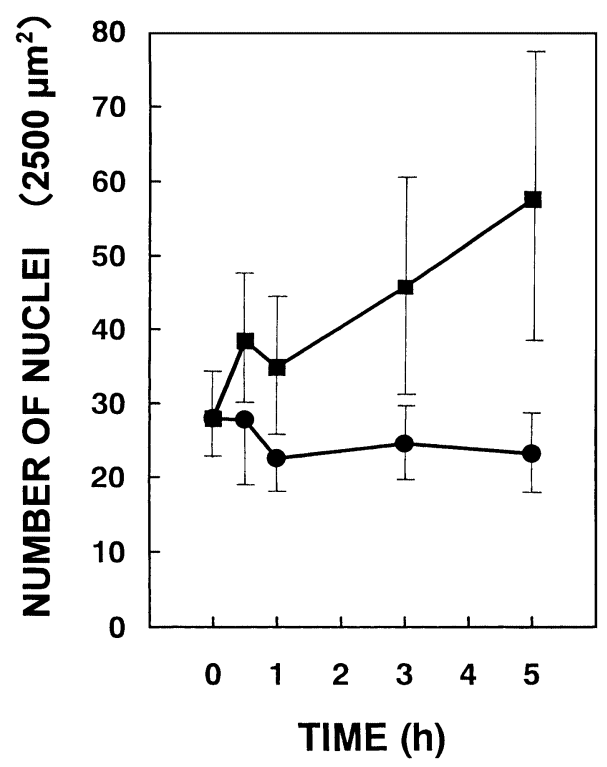

Fig. 9 Time course of accumulation of nuclei in BL-irradiated region. Ordinate: numbers of nuclei counted in randomly selected areas $(50 \times 50 \mu \mathrm{m})$ in the BL-irradiated (squares) and adjacent shaded area (circles) of the fixed, DAPI stained and pressed cell fragment. Data are averages and S.D. from 11-37 measurements. Abscissa: time (h) after the onset of BL ( $\left.456 \mathrm{~nm}, 1.95 \mathrm{~W} \mathrm{~m}^{-2}\right)$. Number of nuclei was counted on pressed double protoplasmic layers. Since cell diameter is approximately $60 \mu \mathrm{m}$, the $50 \times 50 \mu \mathrm{m}$ area corresponds to $53 \%$ of surface area of a 50- $\mu \mathrm{m}$-long cell fragment. Nuclear density of 30 nuclei per unit area is, thus, equivalent to 56 nuclei per $50-\mu \mathrm{m}$-long cylindrical cell; i.e. every $1-\mu \mathrm{m}$-long segment includes one nucleus.

\section{Inhibition of branch induction by APM and CA}

Figure 10 shows the effects of APM, a potent MTdecomposing reagent, and $\mathrm{CA}$, an inhibitor of actomyosinrelated cell motility, on the BL-induced branch induction. Thalli of Vaucheria were pre-incubated for $2 \mathrm{~h}$ with APM or $\mathrm{CA}$, and the percentage of branches induced from the BLirradiated region (about $200 \mu \mathrm{m}$ in width) was counted 7-9 h after the onset of light. Branching was completely inhibited by $100 \mu \mathrm{M}$ APM (Fig. 10a). Among several MT-decomposing reagents so far tested, only APM inhibited the BL-induced branching, although colchicine changed growth polarity at the apical dome (Kataoka 1982) and vinblastine sulfate often caused bursting at the apex. APM probably more easily permeated the cell wall and/or plasmalemma of the proximal region of the cell. The inhibitory effect of APM was reversible, i.e. the branching could be induced $2 \mathrm{~d}$ after thorough washing of the drug.

BL-induced branching was also inhibited by cytochalasins. CA inhibited most effectively among cytochalasins so far tested, i.e. $10 \mu \mathrm{M} \mathrm{CA}$ almost completely inhibited the branching (Fig. 10b), while CB, CC and CD were only effective at concentrations higher than 50-100 $\mu \mathrm{M}$ (data not shown). CA blocked protoplasmic streaming and frequently caused clump- 
a

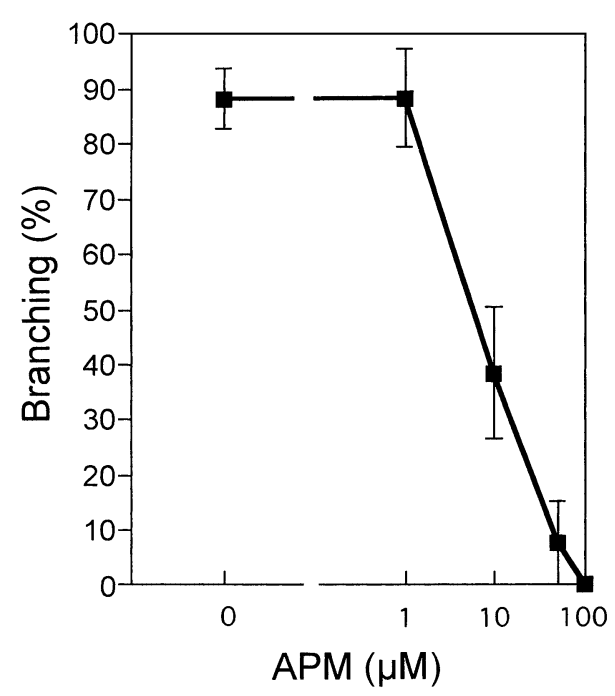

b

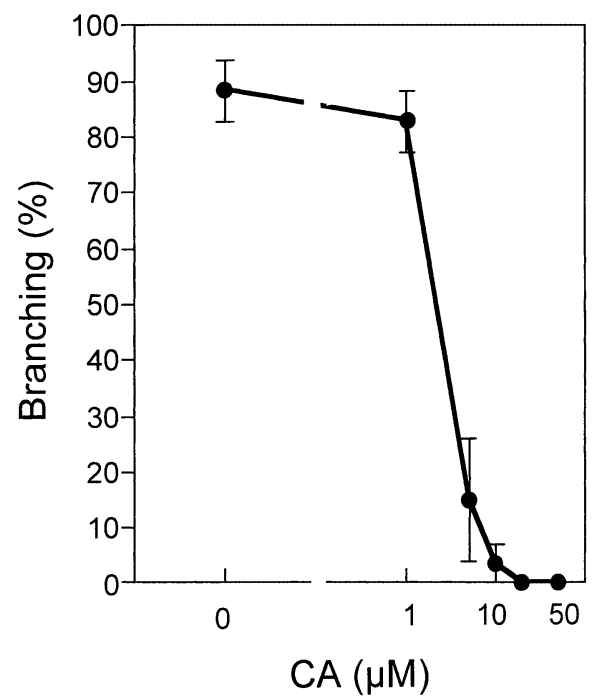

Fig. 10 Dose-response curves for the effects of APM and CA on the BL-induced branching. a, APM; b, CA. After 2 h of pre-incubation with APM or CA at concentrations indicated on abscissa, BL (broad band BL, approximately $7 \mathrm{~W} \mathrm{~m}^{-2}$ ) started to shine about $220 \mu \mathrm{m}$ regions of the cell. Frequency of branch induction was determined 8-12 h after the onset of BL. Data are average and SE from 3-6 repeated experiments.

ing of organelles and plasmolysis at higher concentrations ( $>20 \mu \mathrm{M}$, data not shown). The data shown in Fig. 10b were of course taken from the rest, healthy-looking specimen.

\section{Effects of APM and CA on accumulation of chloroplast and nuclei}

As shown in Fig. 11, the rapid (first) accumulation of chloroplasts in the cortical layer was not inhibited by both APM and CA. Since APM is believed to specifically destroy MTs, this indicates that chloroplasts are not driven by MTrelated motor system. Surprisingly, however, $20 \mu \mathrm{M}$ CA, which completely inhibit the cytoplasmic streaming and branching, permitted the rapid chloroplast accumulation in the BLirradiated region. Approximately $30 \%$ of cells were seriously damaged by $20 \mu \mathrm{M} \mathrm{CA}$ and more than $70 \%$ of cells were killed by $50 \mu \mathrm{M}$ CA. However, even in $50 \mu \mathrm{M}$ CA chloroplasts of the surviving cells could accumulate in BL region. Nevertheless, our result agrees well with the previous study with $\mathrm{CB}$ in $V$. sessilis (Blatt et al. 1980). If CA inhibits only the late starting, long distance migration of chloroplasts, the resistance of chloroplast accumulation to $\mathrm{CA}$ can be understood. Figure 11 clearly demonstrates that both APM and CA inhibit the accumulation of protoplasm into the $\mathrm{BL}$ region. As described before (Fig. 1, 3), the extent of protoplasmic accumulation is photographically judged by the thickness of protoplasm. As demonstrated in Fig. 11b and c, accumulation of protoplasm is not detected.

Two h of incubation with either $100 \mu \mathrm{M}$ APM or $20 \mu \mathrm{M}$ CA sufficiently collapses the MT bundles (Fig. 12). APM $(100 \mu \mathrm{M})$ completely destroyed the MT bundles (Fig. 12c) and concomitantly caused nuclear aggregation (Fig. 12d). This may strongly indicate that the MTs regulate the movement and position of nuclei. CA also destroyed the MT bundles (Fig. 12e), albeit incompletely: a few, fine undulating MT bundles remained $2 \mathrm{~h}$ after the addition of $20 \mu \mathrm{M} \mathrm{CA}$. Although the distribution of nuclei seemed to be unaffected (Fig. 12f), the nuclei totally lost the ability to be transported (see Fig. 11c). This may suggest a possible involvement of actin filaments in maintaining structure and function of the MT bundles. Nevertheless, the data strongly indicate that the accumulation of nuclei and protoplasm that follows the chloroplast accumulation in the cortical cytoplasm is indispensable for the BLinduced branching.

\section{Association of an MT bundle with a nucleus}

To address the question of how nuclei are driven, we examined the structural relationship between nuclei and MT bundles. Superimposing DAPI and MT images on a computer we found that an MT bundle is always associated with a nucleus. Furthermore, using a broadband barrier filter to watch DAPI and MT signal simultaneously, we confirmed that the thick MT bundles are always connected to a nucleus at one end (Fig. 13a, b). By preliminary observation with an electron microscope, we found that one long MT bundle is extending from the centrosome which resides at the apical end of the pyriform nucleus during interphase (Fig. 13c). Beside the forwardly extending thick MT bundle, a short and sparse MT bundle extends behind along the nuclear envelope. The short MT bundles can also be observed with a fluorescence microscope at high magnification. Since Ott (1992) has reported very simi- 


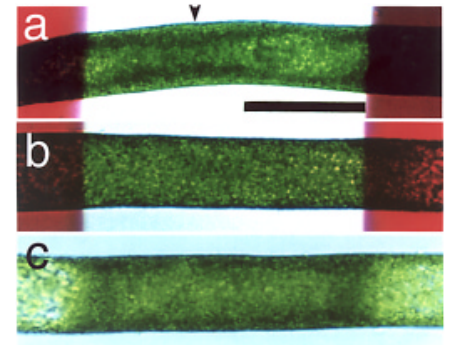

Fig. 11 Chloroplast accumulation in the presence of APM and CA. APM $(100 \mu \mathrm{M})$ and CA $(20 \mu \mathrm{M})$ were added to the bathing solution $2 \mathrm{~h}$ prior to the start of BL partial irradiation. At that time cytoplasmic streaming has already been stopped and under dim red light only weak salutatory movement was detected. Photographs were taken $4 \mathrm{~h}$ after the onset of BL irradiation. (a) Control. Note thick protoplasmic layer in the irradiated region. Chloroplasts sank down from the cortex to the tonoplast area. Arrowhead indicates the presumptive site of branching. A large dumbbell-shaped crystal seen in the central vacuole is probably composed of protein (l. c. Molisch 1926). (b) $100 \mu$ M APM. Chloroplasts are accumulated only in the outermost cytoplasmic layer, but no protoplasm is accumulated. Compare with a: thickness of protoplasm is not increased. (c) $20 \mu \mathrm{M}$ CA. The slit was removed from the optics just before taking this photograph. Chloroplasts are accumulated only near the cell surface. Chloroplasts are more densely accumulated at the both margins than in the middle of the BL region. No significant accumulation of protoplasm is observed. Both APM- and CA-treated cells did not show any further change in morphology by $15 \mathrm{~h}$ under BL partial irradiation, but the chloroplasts gradually dispersed again if $\mathrm{BL}$ light was turned off. Bar $=100 \mu \mathrm{m}$.

lar nucleus-MT bundle complex in the brackish species, $V$. longicaulis, the unique structure seems to be common in the Genus Vaucheria. The parallel increase in number of nuclei and MT bundles (e.g. Fig. 6) and apparent positional similarity between the nuclei and MT bundles (Fig. 7, 8) can now be understood in light of the physical linkage between the nucleus and MT bundle. Accumulation of nuclei in the BL region is no doubt driven by the attached MT bundle and regulated by still unknown MT-associated motor system.

\section{Discussion}

Although the rapid chloroplast accumulation in the cortical layer of the BL-irradiated region seems to be the first and a necessary step for BL-induced branching, it is insufficient by itself. The accumulation of nuclei and surrounding protoplasmic mass in the BL region is indispensable. It seems logical to assume that the accumulated nuclei then express genes of enzymes necessary for softening preexisting cell walls and producing a new growth center. Preliminary experiments with DCMU and actinomycin D, which inhibited branch formation without disturbing the accumulation of chloroplasts and nuclei, may support this. Further detailed pharmacological analyses will be reported elsewhere.
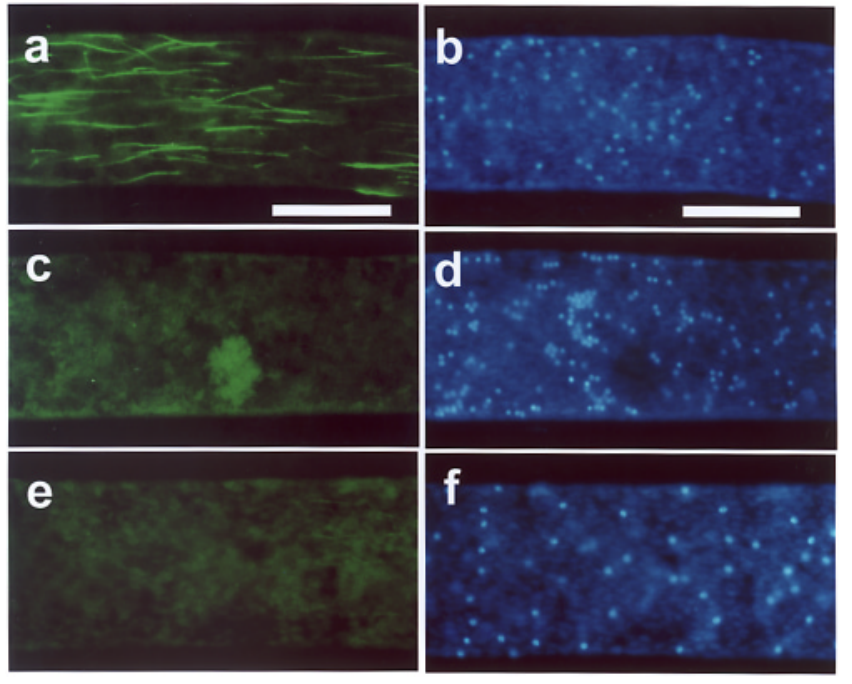

Fig. 12 Effect of APM on MT bundles and nuclear distribution. a, c and e: MT signals. b, d and f: DAPI signals. a and b: Control. $\mathrm{c}$ and d: $2 \mathrm{~h}$ after the addition of $100 \mu \mathrm{M}$ APM. MT bundle disappears and intense green fluorescence probably emitted from monomeric tubulin molecules is seen (c). Nuclei are aggregated to form flocks (d). e and f. $2 \mathrm{~h}$ after the addition of $20 \mu \mathrm{M}$ CA. MT bundles are almost destroyed; only a few fine MT threads is observed (e). Nuclear arrangement is not distorted (f). Bars $=50 \mu \mathrm{m}$.

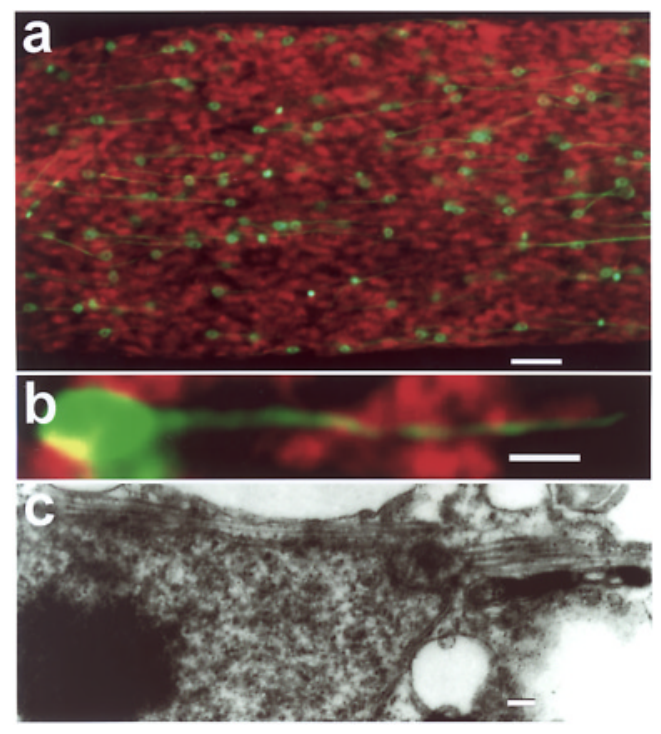

Fig. 13 Structural relationship between nucleus and MT bundle. (a) Nuclei and MTs are simultaneously observed with an epifluorescence microscope equipped with a broadband filter. Note that every MT bundle extends from a single nucleus. Bar $=10 \mu \mathrm{m}$. (b) Enlarged and digitized image of an MT-nucleus complex. Bar $=2 \mu \mathrm{m}$. (c) An electron micrograph of the part of the MT-nucleus complex. Bar $=0.1 \mu \mathrm{m}$. From centrosomal complex two, front and rear, MT bundles are extending. The front bundle corresponds long, thick bundle seen with light microscope. The rear bundle, being along the nucleus, corresponds to obliquely extending, short and fine MT bundles often observed with epifluorescence microscope at high magnification. 


\section{Photoreception}

Nothing is known about photoreceptor(s) and signal transduction pathways that lead to nuclear accumulation. Blatt (1983) determined the action spectrum of chloroplast accumulation in $V$. sessilis. The spectrum having only a single peak at $470 \mathrm{~nm}$ is very different from that for phototropism (Kataoka 1975a), BL-induced apical expansion (Kataoka 1981), and many other flavin-type action spectra. The concomitantly occurring outward (electrogenic) proton current from the BLirradiated region and the chloroplast accumulation may share a common photoreception process (Blatt 1983, Blatt et al. 1981).

However, the current efflux decreased after $1 \mathrm{~h}$ when the chloroplast accumulation was complete (Fig. 1, 5). Branch formation was observed only when a large inward current, which started after cessation of the current efflux, lasted continuously for several hours (Kicherer 1985). Since the time course of the inward current resembles that of nuclear accumulation, photoreceptor system for the accumulation of nuclei and the inward current may be identical but different from that for chloroplast movement.

However, the branch induction may be composed of many other hypothetical BL-dependent responses, such as branching site determination, gene expression and initiation of bulging. Requirement of long duration of irradiation and difficulties in separating these intermediate responses from others impede the determination of action spectra of this photocytomorphogenesis.

\section{Branching-site determination}

The present results provide some information on how the precise position of branching is determined. In Vaucheria cell inward current always occurs at the growing apex or at the presumptive growth center (Kicherer 1985, Kataoka and Weisenseel 1988). The inward current must be carried by ions through ion channels. Emergence of the inward current 2-3 h after the onset of BL (Kicherer 1985) suggests that ion channel proteins for the inward current are expressed and incorporated by this time in the plasmalemma of the presumptive site of branching. Taking into consideration this and the minimum time of $4 \mathrm{~h}$ before the branch initiation (see Fig. 1d, e), the site of the branch initiation is probably determined as early as 2$3 \mathrm{~h}$ after the onset of BL.

Usually, only one branch arises from the center of the BL-irradiated region, if the width of the lighted region is between 50 and $200 \mu \mathrm{m}$ and if the fluence rate of BL is below several $\mathrm{W} \mathrm{m}^{-2}$ (Table 1, Fig. 4; Kataoka 1975b). Since the maximum thickness of protoplasm and the highest density of nuclei are located at the center of the BL region in this condition, concentration of gene products (enzymes) is expected to be highest at the center. Results of the experiments with narrower and/or wedge-shaped BL region (Fig. 4, 5) support this view. When the $\mathrm{BL}$ region was wider than $200 \mu \mathrm{m}, 2-3$ branches were occasionally formed which are sometimes separated from each other by $20-50 \mu \mathrm{m}$. In such cases the amount of nuclei and protoplasm accumulated in the BL region was suffi- cient to develop several branches. By contrast, if the alga was previously kept in darkness for several days, branch induction was not expected, or if any, it was very much delayed (>24 h).

\section{Movement of nuclei by MT}

The direct role of MT bundles in the accumulation of nuclei and protoplasm is strongly supported by the following findings. (1) Orientation of MT bundles and the direction of nuclear movement are parallel to the cell axis. (2) The number of nuclei apparently equals that of long, longitudinally oriented MT bundles (Fig. 6, 8). (3) APM and CA destroys MT bundles and inhibit movement of the nuclei (Fig. 11, 12). Possible involvement of actin in forming and maintaining the MT bundles is considered. (4) APM also destroys the normal distribution pattern of nuclei in the coenocytic tube (Fig. 12). Finally, (5) every nucleus has one long MT bundle ahead (Fig. 13a, b). The MT bundles arise from the centrosome (Fig. 13c, Ott 1992). We preliminarily observed that every nucleus moves forward with the attached MT bundle ahead. Although the molecular mechanism of this nucleus-MT bundle complex is not known yet, an involvement of actomyosin motor system as well as cytoplasmic dynein-like motor protein should be taken into consideration.

Although it is well known that cytochalasins strongly inhibit cytoplasmic streaming in Chara (Williamson 1975, Nagai and Kamiya 1977) and Acetabularia (Nagai and Fukui 1981) as well as in Vaucheria (Blatt et al. 1980), the molecular mechanism of the action of cytochalasins is still obscure. We found that $\mathrm{CA}$ is the most effective among the cytochalasins so far examined. Interestingly, a distinct effectiveness of CA has been reported in some fungi, such as Schizosaccharomyces (Kanbe et al. 1993) or Aspergillus (Torralba et al. 1998), indicating that there might be some phylogenetic relation between Vaucheria and fungi.

Explanation for the ineffectiveness of CA on the chloroplast accumulation in the BL region fails. The molecular mechanism of how chloroplasts are trapped in BL-irradiated region is not fully understood. The simplest explanation currently believed is that the cessation of the cytoplasmic streaming in the BL-irradiated region is the cause of the chloroplast accumulation (Fischer-Arnold 1963). However, since CA inhibits cytoplasmic streaming, tip growth and the branch induction at much lower concentration, cessation of cytoplasmic streaming cannot be the direct cause of the chloroplast accumulation. We demonstrate here that there are two steps in the accumulation of chloroplasts, first $(<1 \mathrm{~h})$ in the cortical cytoplasmic layer and the second $(>1 \mathrm{~h})$ in endoplasm together with nuclei and other organelles. Observed (first) chloroplast accumulation in the presence of CA (Fig. 11c) may indicate that either the "reticulated" actin (Blatt and Briggs 1980b, Blatt et al. 1980) produced by $\mathrm{BL}$ or another CA-resistant actin species drive chloroplasts within the cortical cytoplasmic layer. The mechanism of the newly found chloroplast rotation from face-to profile orientation (Fig. 3) is also not known. 


\section{Ecological significance of the light-induced branching}

Vaucheria grows into a mat in shallow water or on wet soil in a shadowy forest. Fallen leaves frequently cover parts of such mats. The ability to develop branches from openings may be an ecologically profitable trait for such coenocytic algae to expand their habitats. Irradiation of the thallus with narrow BL beam must be a good simulation of the branching occurring in the natural habitat. In other coenocytic algae, such as Caulerpa and Bryopsis a narrow beam of BL can induce similar branching (cf. Kataoka 1975b).

Branching in fern protonemata was experimentally induced by gentle centrifugation (Ootaki 1963, Wada 1995, Wada et al. 1998). Combining centrifugation and red light irradiation after the induction of cell division under white light, Wada (1995) succeeded in inducing branching near the division wall in Adiantum protonema at a high rate. Immediately after the cell division the daughter nuclei moved away from the division wall; but they returned to the base of the branches when the branches began to grow. This indicates that a nucleus does not necessary stay continuously near the site of branching. Instead, a very early step of branch induction and later tip growth require the close localization of the nucleus. This is very different from the case in Vaucheria. Other completely different situations are that a fern protonema cell possesses only one nucleus and that Vaucheria probably lacks a phytochrome system. Nevertheless, the two systems share the important similarity that nuclear control of branching is of short range. Within a coenocytic cell the local photic environment might develop structural or functional differences between irradiated and non-irradiated nuclei. BL-irradiated nuclei may not only serve as leaders in cytodifferentiation but also influence their neighbors. In coenocytic cells, such functional and structural differentiation among nuclei can also be expected during the development of apico-basal polarity.

\section{Acknowledgements}

This study was partly supported by a Grant-in-Aids for Scientific Research (no. 08304045) from the Ministry of Education, Science, Sports and Culture, Japan (Mombusho) and a grant from Takeda Science Foundation (to HK, 1997). We are grateful to Nihon Bayer Agrochem for the kind donation of APM. We wish to thank Dr. Taizo Motomura of Hokkaido University for the use of his fluorescence microscope equipped with broad-band filter and his critical and valuable discussion. We are also grateful to Prof. Tamotsu Ootaki of our institute for his helpful advice.

\section{References}

Aya, T., Takase, I., Kishino, S. and Kurihara, K. (1975) Amiprophos-Methyl, a new herbicide in upland crops. In Proceedings of the Fifth Asian-Pacific Weed Science Society Conference. pp. 138-141. The Asian-Pacific Weed Science Society, Tokyo.

Blatt, M.R. (1983) The action spectrum for the chloroplast movements and evidence for blue-light-photoreceptor cycling in the alga Vaucheria. Planta 159: 267-276.

Blatt, M.R. and Briggs, W.R. (1980a) Blue-light stimulates a local electric cur- rent efflux in the alga Vaucheria sessilis. Carnegie Inst. Wash. Year Book 1979: 123-125.

Blatt, M.R. and Briggs, W.R. (1980b) Blue-light-induced cortical fiber reticulation concomitant with chloroplast aggregation in the alga Vaucheria sessilis. Planta 147: 355-362.

Blatt, M.R., Wessels, N.K. and Briggs, W.R. (1980) Actin and cortical fiber reticulation in the siphonaceaous alga Vaucheria sessilis. Planta 147: 363375.

Blatt, M.R., Weisenseel, M.H. and Haupt, W. (1981) A light-dependent current associated with chloroplast aggregation in the alga Vaucheria sessilis. Planta 152: 513-526.

Fischer-Arnold, G. (1963) Untersuchungen über die Chloroplasten-bewegung bei Vaucheria sessilis. Protoplasma 56: 495-520.

Haupt, W. (1963) Photoreceptorprobleme der Chloroplastenbewegung. Ber. Dtsch. Bot. Ges. 76: 313-322.

Haupt, W. (1965) Reception of environmental stimuli orienting growth and movement in lower plants. Annu. Rev. Plant Physiol. 16: 267-290.

Haupt, W. and Schönbohm, E. (1970) Light-oriented chloroplast movement. In Photobiology of Microorganisms. Edited by Halldal, P. pp. 283-307. WileyInterscience, London, New York, Sydney, Toronto.

Kadota, A. and Wada, M. (1992) Reorganization of the cortical cytoskeleton in tip-growing fern protonemal cells during phytochrome-mediated phototropism and blue light-induced apical swelling. Protoplasma 166: 35-41.

Kanbe, T., Akashi, T. and Tanaka, K. (1993) Effect of cytochalasin A on actin distribution in the fission yeast Schizosaccharomyces pombe studies by fluorescent and electron microscopy. Protoplasma 176: 24-32.

Kataoka, H. (1975a) Phototropism in Vaucheria geminata I. The action spectrum. Plant Cell Physiol. 16: 427-437.

Kataoka, H. (1975b) Phototropism in Vaucheria geminata II. The mechanism of bending and branching. Plant Cell Physiol. 16: 439-448.

Kataoka, H. (1980) Phototropism: determination of an action spectrum in a tipgrowing cell. In Handbook of Phycological Methods. Developmental \& Cytological Methods. Edited by Gantt, E. pp. 205-218. Cambridge University Press, Cambridge.

Kataoka, H. (1981) Expansion of Vaucheria cell apex caused by blue or red light. Plant Cell Physiol. 22: 583-595.

Kataoka, H. (1982) Colchicine-induced expansion of Vaucheria cell apex. Alteration from isotropic to transversally anisotropic growth. Bot. Mag. Tokyo 95: 317-330.

Kataoka, H. (1987) The light-growth response of Vaucheria. A conditio sine qua non of the phototropic response? Plant Cell Physiol. 28: 61-71.

Kataoka, H. (1988) Negative phototropism in Vaucheria terrestris regulated by calcium I. Dependence on background blue light and external Ca-concentration. Plant Cell Physiol. 29: 1323-1330.

Kataoka, H. (1990) Negative phototropism of Vaucheria terrestris regulated by calcium II. Inhibition by $\mathrm{Ca}^{2+}$-channel blockers and mimesis by A23187. Plant Cell Physiol. 31: 933-940.

Kataoka, H. and Watanabe, M. (1993) Negative phototropism of Vaucheria terrestris regulated by calcium III. The role of calcium characterized by use of a high-power argon-ion laser as the source of unilateral blue light. Plant Cell Physiol. 34: 737-744.

Kataoka, H. and Weisenseel, M.H. (1988) Blue light promotes ionic current influx at the growing apex of Vaucheria terrestris. Planta 173: 490-499.

Kicherer, R. (1985) Endogene und Blaulicht-induzierte Ionenströme bei der Alge Vaucheria sessilis. p. 59. Dissertation to Universität Erlangen-Nürnberg.

Kiermayer, O. (1981) Cytoplasmic basis of morphogenesis in Micrasterias. In Cytomorphogenesis in Plants. Edited by Kirmayer, O. pp. 147-189. Springer Verlag, Wien, New York.

Maekawa, T. and Nagai, R. (1988) Reorganization of microtubule bundles in Dichotomosiphon: its implication in the light-induced translocation of cytoplasm. Protoplasma [suppl. 1]: 162-171.

Molisch, H. (1926) Botanische Beobachtungen in Japan IV. Über das massenhafte Vorkommen von Eiweissspindeln in einer Vaucheria. Sci. Rep. Tohoku Univ. Jpn. IV Biol. 1: 105-109.

Nagai, R. and Fukui, S. (1981) Differential treatment of Acetabularia with cytochalasin $\mathrm{B}$ and $\mathrm{N}$-ethylmaleimide with special reference to their effects on cytoplasmic streaming. Protoplasma 109: 79-89.

Nagai, R. and Kamiya, N. (1977) Differential treatment of chara cells with cytochalasin B with special reference to its effect on cytoplasmic streaming. Exp. Cell Res. 108: 231-237. 
Nagata, Y. (1973) Rhizoid differentiation in Spirogyra I. Basic feature of rhizoid formation. Plant Cell Physiol. 14: 531-541.

Ootaki, T. (1963) Modification of the developmental axis by centrifugation in Pteris vittata. Cytologia 28: 21-29.

Ott, D.W. (1992) The role of the cytoskeleton in organelle translocation in Vaucheria longicaulis. In The Cytoskeleton of the Algae. Edited by Menzel, D.M. pp. 255-272. CRC Press, Boca Raton, Ann Arbor, London, Tokyo.

Reynolds, E.S. (1963) The use of lead citrate at high $\mathrm{pH}$ as an electron-opaque stain in electron microscopy. J. Cell Biol. 17: 208-212.

Riquelme, M., Reynaga-Peña, C.G., Gierz, G. and Bartnicki-García, S. (1998) What determines growth direction in fungal hyphae? Fungal Gen. Biol. 24: 101-109.

Senn, G. (1908) Die Gestalts- und Lageveränderung der Pflanzen-
Chromatophoren. p. 397. Verlag von Wilhelm Engelmann, Leipzig.

Spurr, A.R. (1969) A low viscosity epoxy resin embedding for electron microscopy. J. Ultrastruct. Res. 26c: 31-43.

Torralba, S., Raudaskoski, M., Pedregosa, A.M. and Laborda, F. (1998) Effect of cytochalasin A on apical growth, action cytoskeleton organization and enzyme secretion in Aspergillus nidrans. Microbiology 144: 45-53.

Wada, M. (1995) Nuclear behavior during branch formation in a centrifuged Adiantum protonema and the nuclear polarity. J. Plant Res. 108: 501-509.

Wada, M., Nozue, K. and Kadota, A. (1998) Cytoskeletal pattern changes during branch formation in a centrifuged Adiantum protonema. J. Plant Res. 111: 53-58.

Williamson, R. (1975) Cytoplasmic streaming in Chara: a cell model activated by ATP and inhibited by cytochalasin B. J. Cell Biol. 17: 655-668.

(Received September 27, 2000; Accepted December 8, 2000) 